\title{
Rheometer mit zwei EC-Motoren
}

A nton Paar hat ein neues Rheometer entwickelt, das auf der sogenannten TwinDrive-Technologie beruht und erstmals Messungen im Nanometerbereich möglich macht.

Die TwinDrive-Technologie verbindet zwei elektronisch kommutierte Motoren in einem modularen Setup: Der obere EC-Motor ist fest integriert, der untere kann nach Belieben aus- und eingebaut werden. Damit besteht dank hoher Regeldynamik die Möglichkeit, auch schwierige Messaufgaben mit höchster Präzision zu lösen. Sind beide Motoren im Antriebs- und Messmodus aktiv, erweitern sich die Testmöglichkeiten - z. B. mit einer optionalen Gegenrotation für die erweiterte Rheo-Mikroskopie. Außerdem steigt die Messgenauigkeit. Ein weiterer Vorteil der Kombination zweier Motoren ist die doppelte maximale Scherrate, die für rheologische Tests eingestellt werden kann. Optional kann das neue Rheometer auch für dehnrheologische Anwendungen oder Versuche mit Parallelrotation bei verschiedenen Geschwindigkeiten eingesetzt werden.
Je nach Anwendung und Anforderung lässt sich die untere Motoreinheit leicht entfernen - ein Vorgang, der von einem patentierten System zur automatischen Erkennung von Messsystemen voll unterstützt wird. Dieser Modus ermöglicht die Nutzung der großen Bandbreite an passenden Temperiereinheiten (von minus $150{ }^{\circ} \mathrm{C}$ bis $1000{ }^{\circ} \mathrm{C}$ ) und Drücken (von Vakuum bis 1000 bar) sowie von applikationsspezifischem $\mathrm{Zu}$ behör.

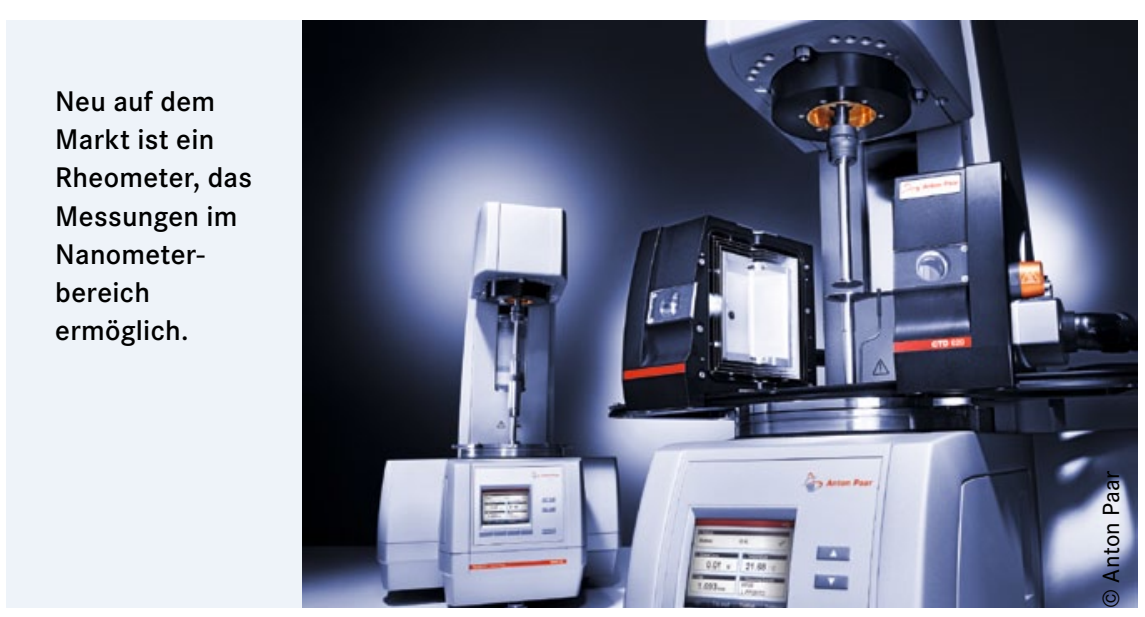

\section{Trocken geklebte Isolierglaskonstruktionen}

$D^{a}$ ank der Weiterentwicklung von solierglaskonstruktionen und deren Verklebung lassen sich heute auch großzügig verglaste Gebäude mit energiesparendem Bauen vereinbaren. Im Vordergrund steht hier ein Verfahren zur Trockenverklebung und die dadurch gegebene Designfreiheit im Fensterbau.

„Dry Static Bonding“ von Lohmann basiert auf einer Isolierglasverklebung, bei der die Glasscheibe mit einem HighEnd-Klebeband direkt in den Fensterflügel geklebt wird. Dabei sorgt das doppelseitige Montageklebeband für Stabilität der Profile und dichtet zuverlässig gegen Kälte. Aufgrund der hohen Endfestigkeit und der Alterungsbeständigkeit des Klebebandes bleibt die geklebte Rahmenkon- struktion stabil und gewährleistet eine sichere Lastabtragung. Ein stabilisierender Stahlkern innerhalb des Fensterprofils wird somit hinfällig - der Hersteller spart Material- und Fertigungskosten. Ein weiterer Vorteil: Durch den Wegfall der Stahlarmierung wird das Gewicht des Fensters reduziert und die freigewordene Profilkammer kann mit zusätzlichen Dämmstoffen ausgestattet werden. Wärmebrücken sind somit eliminiert und die Wärmedämmung des Fensters optimiert. Auch in Bezug auf Einbruchschutz gewinnt die Fensterkonstruktion an Sicherheit, denn durch die umlaufende Klebfuge am Glasrand wird das Aufhebeln der Scheibe erschwert. Der Profilhersteller kann das Klebeband direkt auf das Fens-
Dieses neue Rheometer ermöglicht z. B. Messungen bei niedrigen Drehmomenten bis $\mathrm{zu} 1 \mathrm{nNm}$ und die Kontrolle von Winkelabweichungen bis zu 100 nrad. Diese Präzisionsleistung betrifft auch die Messspaltkontrolle des Systems: Ein neuer Piezo-Flansch schafft Spaltgrößenänderungen bis zu $10 \mathrm{~nm}$.

Weitere Infos: Anton Paar $\mathrm{GmbH}$, Weitere Infos: Anton Paar GmbH,

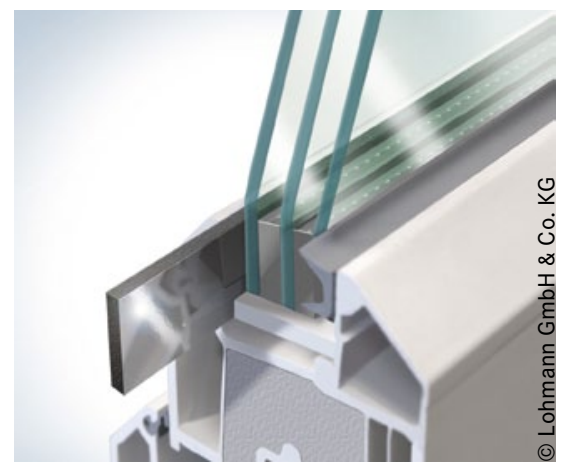

terprofil applizieren oder es als Adapterprofil an das bestehende Profil anfügen. Beide Möglichkeiten lassen sich in bestehende Prozesse integrieren, ohne in neue Maschinen zu investieren.

Weitere Infos: Lohmann GmbH \& Co. KG, 56567 Neuwied, info@lohmann-tapes.com, www.lohmann-tapes.com 